\title{
Configuration Spaces of Mechanical Linkages
}

\author{
D. Jordan ${ }^{1}$ and M. Steiner ${ }^{2}$ \\ ${ }^{1}$ Mathematisches Institut, Universität Bern, \\ CH-3012 Bern, Switzerland \\ jordan@math-stat.unibe.ch \\ 2 Département de Mathématiques, EPF Lausanne, \\ CH-1015 Lausanne, Switzerland \\ marcel.steiner@epfl.ch
}

\begin{abstract}
We present an easy to survey constructive method using only basic mathematics which allows us to define a homeomorphism between any compact real algebraic variety and some components of the configuration space of a mechanical linkage. The aim is to imitate addition and multiplication in the framework of weighted graphs in the euclidean plane that permit a "mechanical description" of polynomial functions, and thus of varieties.
\end{abstract}

\section{Introduction}

A mechanical linkage $\mathcal{G}$ is a mechanism in the euclidean plane $\mathbb{R}^{2}$ that is built up exclusively from rigid bars joined along flexible links. Some links of the linkage may be pinned down with respect to a fixed frame of reference. The configuration space [G] of a mechanical linkage is the totality of all its admissible positions in the euclidean plane.

Configuration spaces of such linkages have been studied for centuries as one of the basic topics of kinematics, and it is a known fact that their configuration spaces are compact real algebraic varieties naturally embedded in $\left(\mathbb{R}^{2}\right)^{n}$, where $n$ is the number of vertices in the graph. Therefore it is natural to ask whether, conversely, every compact real algebraic variety arises as the configuration space of some mechanical linkage in the euclidean plane. In [9] Lebesgue gives an account of several results, including Kempe's universality theorem, not for the configuration space of the mechanism itself, but for the orbit of one of its vertices: "Toute courbe algébrique peut être tracée à l'aide d'un système articulé." The existence of the following universality theorem for some components of the configuration space has been part of folklore for at least two decades. 
Theorem 1.1. Let $V \subset \mathbb{R}^{q}$ be a compact real algebraic variety with the topology induced by the euclidean metric of $\mathbb{R}^{q}$. Then $V$ is homeomorphic to some components $\bigsqcup_{j \in I \subset J} T_{j}$ of the configuration space $[\mathcal{H}]=\bigsqcup_{j \in J} T_{j}$ of a mechanical linkage $\mathcal{H}$, where $T_{j}, j \in J$, are the components of $[\mathcal{H}]$.

Reference [5] which was circulated as a preprint in early 1997 already contains the main idea of the present proof of Theorem 1.1, but some subtleties were neglected there. In contrast to the preprint [8] of Kapovich and Millson we give an explicit construction of the mechanical linkage using only basic mathematics.

Notice that, in the past, a number of similar universality properties have been established, beginning with Mnëv's celebrated theorem about oriented matroids [10], and leading to Richter-Gebert's lucid kinematic studies of four-dimensional convex polytopes [11].

\section{Preliminaries}

\subsection{Definitions}

The first step is to replace our intuitive idea of a mechanical linkage by an exact mathematical definition:

Definition 2.1. The triple $\mathcal{G}=(V, E, d)$ consisting of

(1) a set of vertices $V=V_{\text {fix }} \cup V_{\text {free }}$, with $V_{\text {fix }}=\left\{V_{1}, \ldots, V_{m}\right\}$ and $V_{\text {free }}=\left\{V_{m+1}, \ldots\right.$, $\left.V_{n}\right\}$

(2) a set of edges $E=\left\{\left\{V_{i_{1}}, V_{j_{1}}\right\},\left\{V_{i_{2}}, V_{j_{2}}\right\}, \ldots,\left\{V_{i_{k}}, V_{j_{k}}\right\}\right\}$ with $i_{l}, j_{l} \in\{1, \ldots, n\}$, $i_{l} \neq j_{l}$, such that any two vertices in $V$ are connected by a sequence of elements of $E$, and

(3) a weight function $d: E \rightarrow \mathbb{R}_{+}$, that attaches to every edge $\left\{V_{i_{l}}, V_{j_{l}}\right\}$ in $E$ a length (weight) $d\left(V_{i_{l}}, V_{j_{l}}\right) \in \mathbb{R}_{+}$,

is called a connected weighted graph.

Definition 2.2. Let $\mathcal{G}=(V, E, d)$ be a connected weighted graph.

(1) The graph $\mathcal{G}$ is called a mechanical linkage if $\mathcal{G}$ is realizable in $\mathbb{R}^{2}$, i.e., if a mapping $\varphi: V \rightarrow \mathbb{R}^{2}$ exists, such that $d_{\mathbb{R}}\left(\varphi\left(V_{i}\right), \varphi\left(V_{j}\right)\right)=d\left(V_{i}, V_{j}\right)$ for all $\left\{V_{i}, V_{j}\right\} \in E$, where $d_{\mathbb{R}}$ is the euclidean metric in $\mathbb{R}^{2}$.

(2) A realization $\xi$ of $\mathcal{G}=(V, E, d)$ is a set $\left\{\varphi_{\xi}\left(V_{1}\right), \ldots, \varphi_{\xi}\left(V_{n}\right)\right\}$ of points in $\mathbb{R}^{2}$ such that $d_{\mathbb{R}}\left(\varphi_{\xi}\left(V_{i}\right), \varphi_{\xi}\left(V_{j}\right)\right)=d\left(V_{i}, V_{j}\right)$ for all $\left\{V_{i}, V_{j}\right\} \in E$.

We often write $V_{j}$ at $p \in \mathbb{R}^{2}$ instead of $\varphi\left(V_{j}\right)=p \in \mathbb{R}^{2}$, i.e., consider $V_{j}$ as $\varphi\left(V_{j}\right)$. Notice that there are connected weighted graphs with only three vertices that are not realizable in the euclidean plane and obviously a realization of a mechanical linkage is in general not unique. We define the configuration space of a mechanical linkage as a subset of $\mathbb{R}^{2 n}$, which obtains in natural way a topological structure: 
Definition 2.3. Let $\mathcal{G}=(V, E, d)$ be a mechanical linkage and let $\left\{p_{1}, \ldots, p_{m}\right\}$ be fixed points in $\mathbb{R}^{2}$ with $m \geq 2$, such that $d_{\mathbb{R}}\left(p_{i}, p_{j}\right)=d\left(V_{i}, V_{j}\right)$ for all $\left\{V_{i}, V_{j}\right\} \in E$ with $V_{i}, V_{j} \in\left\{V_{1}, \ldots, V_{m}\right\}=V_{\text {fix }}$. Then the configuration space of $\mathcal{G}$ is defined by

$$
\begin{aligned}
{[\mathcal{G}]:=} & \left\{\xi \text { realization of } \mathcal{G} ; \varphi_{\xi}\left(V_{j}\right)=p_{j}, \forall V_{j} \in V_{\text {fix }}\right\} \\
= & \left\{\left(x_{1}, \ldots, x_{n}\right) \in\left(\mathbb{R}^{2}\right)^{n} ; x_{j}=p_{j}, \forall j \in\{1, \ldots, m\}\right. \text { and } \\
& \left.d_{\mathbb{R}}\left(x_{i}, x_{j}\right)=d\left(V_{i}, V_{j}\right), \forall\left\{V_{i}, V_{j}\right\} \in E\right\}
\end{aligned}
$$

with the topology induced by the euclidean metric of $\mathbb{R}^{2 n}$.

Remark 2.4. If $M$ and $N$ are topological spaces, then $M U N$ denotes the disjoint topological sum of the two spaces.

Often we are only interested in subspaces of $[\mathcal{G}]$ for a given mechanical linkage $\mathcal{G}=$ $(V, E, d)$ : restricting a set of vertices $\left\{V_{j 1}, \ldots, V_{j k}\right\} \subseteq V$ in a subset $Y \subset \mathbb{R}^{2 k}$ allows us to consider $\left\{\xi \in[\mathcal{G}] ;\left(V_{j 1}, \ldots, V_{j k}\right)\right.$ in $\left.Y\right\}:=\left\{\xi \in[\mathcal{G}] ;\left(\varphi_{\xi}\left(V_{j 1}\right), \ldots, \varphi_{\xi}\left(V_{j k}\right)\right) \in\right.$ $Y\} \subseteq[\mathcal{G}]$.

Every realization of a mechanical linkage can be represented by a planar drawing consisting of a set of points $V$ and a set of straight lines $E$ with the prescribed length $d$ : an empty small circle is a vertex pinned down at a point in the plane, i.e., an element of $V_{\text {fix }}$ and a filled circle is an element of $V_{\text {free }}$. This enables us to speak in a natural way of motions of vertices and of rigid mechanical linkages:

Definition 2.5. $\quad$ Let $\mathcal{G}=(V, E, d)$ be any mechanical linkage.

(1) Let $Y \subset \mathbb{R}^{2 k}, Y \neq p t$, be connected. The $k$-tuple $\left(V_{j 1}, \ldots, V_{j k}\right)$ of vertices of $V$ can be moved on $Y$ if there exists a component $C$ of $\left\{\xi \in[\mathcal{G}] ;\left(\varphi_{\xi}\left(V_{j 1}\right), \ldots\right.\right.$, $\left.\left.\varphi_{\xi}\left(V_{j k}\right)\right) \in Y\right\}$ with $\left\{\left(\varphi_{\xi}\left(V_{j 1}\right), \ldots, \varphi_{\xi}\left(V_{j k}\right)\right) ; \xi \in C\right\}=Y$.

(2) The vertex $V_{j}$ is called rigid, with respect to a realization $\xi \in[\mathcal{G}]$ with $\varphi_{\xi}\left(V_{j}\right)=p$, if an open neighborhood $U \subseteq[\mathcal{G}]$ of $\xi$ exists, such that for any realization $\tilde{\xi} \in U$ it follows that $\varphi_{\tilde{\xi}}\left(V_{j}\right)=p$.

(3) A realization $\xi \in[\mathcal{G}]$ of a mechanical linkage $\mathcal{G}$ is called rigid if every vertex of $\mathcal{G}$ is rigid with respect to $\xi$, i.e., $\xi$ is an isolated point of $[\mathcal{G}]$.

(4) A realization $\xi \in[\mathcal{G}]$ of a mechanical linkage $\mathcal{G}$ is called regular if there is $\operatorname{dim} \operatorname{aff}\left\{\varphi_{\xi}\left(V_{k}\right), \varphi_{\xi}\left(V_{l}\right), \varphi_{\xi}\left(V_{m}\right)\right\}=2$ for all $\left\{V_{k}, V_{l}\right\},\left\{V_{l}, V_{m}\right\} \in E,\left\{V_{k}, V_{m}\right\} \notin$ $E$ with $V_{l} \in V_{\text {free }}, k \neq m$.

Each of the mechanical linkages used below has a special area of effectiveness in the present constructive proof. The reader may imagine that not all realizations will do the job: consider the mechanical linkage $\mathcal{K}$ in Section 2.3.3, we remark that apart from the drawn realization in Fig. 4 others are looking quite different and may appear by $\mathbb{Z}_{2}$-actions on some vertices of $\mathcal{K}$. There we show that under special conditions $X$ can move only on a straight line orthogonal to $O A$. Exactly those realizations are used in the present construction, which satisfies the prescribed claims, e.g., in Fig. 4 the vertex $X$ lies in an orthogonal line to $O A$, and $\mathcal{K}$ has one degree of freedom only. In general, under 
additional conditions a motion of vertices allows us to parameterize those realizations obtained by the motion, which are distinct from others by $\mathbb{Z}_{2}$-actions:

Lemma 2.6. Let $\mathcal{G}=(V, E, d)$ be any mechanical linkage and $V_{j l} \in V, \forall l \in$ $\{1, \ldots, k\}$, such that departing from $\xi_{0} \in[\mathcal{G}]$ the $k$-tuple $\left(V_{j 1}, \ldots, V_{j k}\right)$ can be moved on the connected subset $Y \subset \mathbb{R}^{2 k}$. If each obtained realization $\xi_{p} \in[\mathcal{G}]$ with $\left(V_{j 1}, \ldots, V_{j k}\right)$ fixed at $p \in Y$ (i.e., $V_{j 1}, \ldots, V_{j k} \in V_{\text {fix }}$ for the moment) is (i) rigid and (ii) regular, then

$$
\left\{\xi \in[\mathcal{G}] ;\left(\varphi_{\xi}\left(V_{j 1}\right), \ldots, \varphi_{\xi}\left(V_{j k}\right)\right) \in Y\right\} \approx Y \amalg T_{\text {rest }},
$$

where $T_{\text {rest }}$ is any subspace of $[\mathcal{G}]$. The motion of $\left(V_{j 1}, \ldots, V_{j k}\right)$ on $Y$ is then called parameterizing.

Proof. (i) implies that a motion of any tuple of vertices in $V-\left\{V_{j 1}, \ldots, V_{j k}\right\}$ is only possible by a motion of $\left(V_{j 1}, \ldots, V_{j k}\right)$. (ii) induces that this motion is unique for all vertices in $V$ : during the motion any realization other than $\xi_{p}$ is given by a $\mathbb{Z}-2$-action on a vertex, if $\left(V_{j 1}, \ldots, V_{j k}\right)$ is assumed to be fixed at $p$. This ensures that $T_{\text {rest }}$ is not connected to a component $Y^{\prime}$ in $\left\{\xi \in[\mathcal{G}] ;\left(\varphi_{\xi}\left(V_{j 1}\right), \ldots, \varphi_{\xi}\left(V_{j k}\right)\right) \in Y\right\}$. The position of the vertices $V-\left\{V_{j 1}, \ldots, V_{j k}\right\}$ are parameterized by trigonometric polynomials of the variable $\left(\varphi_{\xi}\left(V_{j 1}\right), \ldots, \varphi_{\xi}\left(V_{j k}\right)\right) \in Y$, therefore $Y^{\prime} \approx Y$.

Notice that condition (ii) is too strong to obtain the result of Lemma 2.6 but it is sufficient to handle special mechanical linkages used in Sections 2.3 and 3. The drawn realizations there are called starting realizations $\xi_{0}$. We often describe natural realizations by a parameterizing motion of a set of vertices departing from the starting realization.

\subsection{Real Algebraic Geometry}

First we recall some facts about real algebraic geometry that serve as a basis for our considerations.

Definition 2.7. Let $A$ be a subset of $\mathbb{R}\left[X_{1}, \ldots, X_{q}\right]$. The set $\mathcal{L}(A):=\left\{x \in \mathbb{R}^{q} ; f(x)=\right.$ $0, \forall f \in A\}$ is called the set of zeros of $A$. Let $S$ be a subset of $\mathbb{R}^{q}$. We note $\mathcal{F}(S):=$ $\left\{f \in \mathbb{R}\left[X_{1}, \ldots, X_{q}\right] ; \forall x \in S, f(x)=0\right\}$. The real algebraic varieties are those subsets $V$ of $\mathbb{R}^{q}$, for which $\mathcal{L}(\mathcal{F}(V))=V$.

Since $\mathbb{R}$ is a Noetherian ring, we can use Hilbert's Basis Theorem to show that every ideal in $\mathbb{R}\left[X_{1}, \ldots, X_{q}\right]$ is finitely generated. Every evaluation of a polynomial in $A$ on $\mathbb{R}^{q}$ is real, thus $V$ in $\mathbb{R}^{q}$ can always be described as the zero set of a single polynomial $F \in \mathbb{R}\left[X_{1}, \ldots, X_{q}\right]$ :

Lemma 2.8. For each real algebraic variety $V \subseteq \mathbb{R}^{q}$ a polynomial $F \in \mathbb{R}\left[X_{1}, \ldots, X_{q}\right]$ exists such that $V=\mathcal{L}(F)$.

Proof. Define $F:=f_{1}^{2}+\cdots+f_{l}^{2}$, where $f_{1}, \ldots, f_{l}$ are generators of the ideal $\mathcal{F}(V)$. 


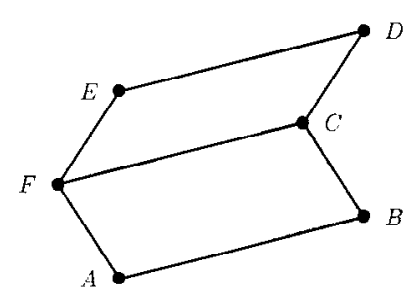

Fig. 1. Translation linkage $\mathcal{T}$.

\subsection{Special Mechanical Linkages}

The proof of the main theorem builds on knowledge about the following three basic mechanical linkages, see [9].

2.3.1. Translation Linkage $\mathcal{T}$. The translation linkage $\mathcal{T}$ is the mechanical linkage shown in Fig. 1, which satisfies the conditions

(a) $d(A, B)=d(C, F)=d(D, E) \in \mathbb{R}_{+}^{*}$,

(b) $d(A, F)=d(B, C) \in \mathbb{R}_{+}^{*}$, and

(c) $d(E, F)=d(C, D) \in \mathbb{R}_{+}^{*}$.

In the proof of Theorem 1.1 the translation linkage appears as a part of the addition and multiplication linkage. There we use only regular realizations of $\mathcal{T}$ such that $A B$, $F C$, and $E D$ are parallel. Note that without any risk of confusion the denotations of the vertices are often changed, since we have to couple together several translation linkages.

2.3.2. Conformal Linkage $\mathcal{C}$. The conformal linkage $\mathcal{C}$ is the mechanical linkage shown in Fig. 2 which satisfies the conditions

(a) $d(O, B)=d(O, D)$ and $d(B, C)=d(C, D) \in \mathbb{R}_{+}^{*}$,

(b) $d\left(O, B^{\prime}\right)=d\left(O, D^{\prime}\right)$ and $d\left(B^{\prime}, C^{\prime}\right)=d\left(C^{\prime}, D^{\prime}\right) \in \mathbb{R}_{+}^{*}$,

(c) $d\left(A, A^{\prime}\right)=d\left(E, E^{\prime}\right) \in \mathbb{R}_{+}$with $d(O, A)=d(O, E)=\frac{1}{2} d(O, B)$ and $d\left(O, A^{\prime}\right)=d\left(O, E^{\prime}\right)=\frac{1}{2} d\left(O, B^{\prime}\right)$, and

(d) the kite quadrilaterals $O B C D$ and $O B^{\prime} C^{\prime} D^{\prime}$ are similar for all natural realizations.

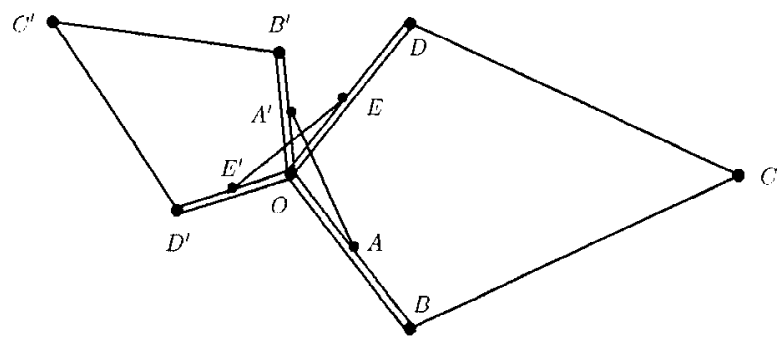

Fig. 2. Conformal linkage $\mathcal{C}$. 


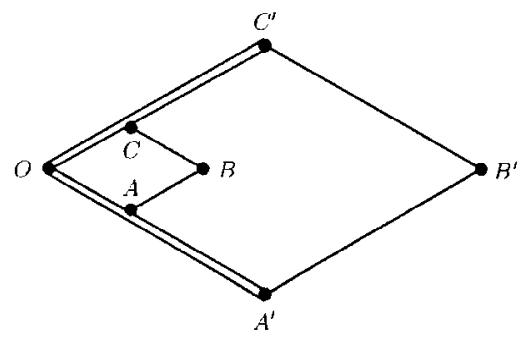

Fig. 3. Linear linkage $\mathcal{L}$.

In the proof of Theorem 1.1 the conformal linkage appears as a part of the multiplication linkage. There we use only regular realizations of $\mathcal{C}$, especially $C$ and $O$ never lie at the same point, or the vertices $O, B, C, D$ lie in one line.

2.3.3. Linear Linkage $\mathcal{L}$. The linear linkage $\mathcal{L}$ is the mechanical linkage shown in Fig. 3, a special case of $\mathcal{C}$. It satisfies the conditions

(a) $d(O, A)=d(O, C)=d(A, B)=d(B, C) \in \mathbb{R}_{+}^{*}$,

(b) $d\left(O, A^{\prime}\right)=d\left(O, C^{\prime}\right)=d\left(A^{\prime}, B^{\prime}\right)=d\left(B^{\prime}, C^{\prime}\right)=\lambda \cdot d(O, A)$ with $\lambda>1$, and

(c) the rhombuses $O A B C$ and $O A^{\prime} B^{\prime} C^{\prime}$ are similar for all natural realizations.

In the proof of Theorem 1.1 the linear linkage appears as a part of the multiplication linkage. There we use only regular realizations of $\mathcal{L}$, especially $B$ and $O$ never lie at the same point, or the vertices $O, A, B, C$ lie in one line. Note that we use $\mathcal{L}$ changing the denotations of the vertices below.

2.3.4. Kempe's Linkage $\mathcal{K}$. We consider the mechanical linkage $\mathcal{K}$ in Fig. 4 which is defined in [9] as sextilatère de Kempe. It satisfies the properties

(a) $d(O, E)=\sqrt{2} / 2 \cdot d(O, A) \in \mathbb{R}_{+}^{*}$,

(b) for all natural realizations $O A B D$ is a rhombus, and

(c) for all natural realizations $O E B D$ and $C X E B$ are similar kite quadrilaterals.

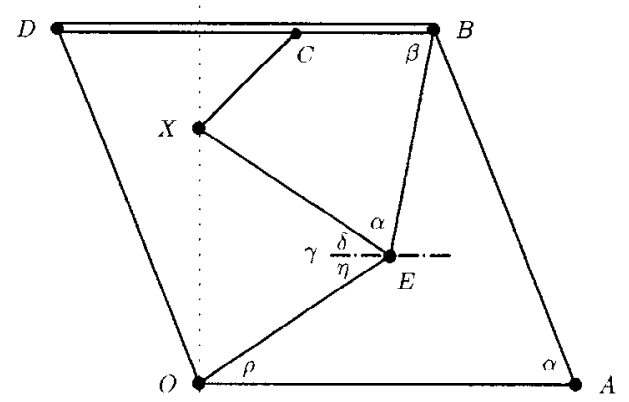

Fig. 4. Starting realization $\xi_{0}$ of Kempe's linkage $\mathcal{K}$. 
Our interest lies in a subset of the configuration space of $\mathcal{K}$, and we chose $O, A \in V_{\text {fix }}$ for the moment. In the point $O$ we define a local orthogonal system of coordinates of $\mathbb{C}$, such that $O$ is pinned down at the origin of $\mathbb{C}$ and $A$ at $d(O, A)$ of the positive real axis. Note that in the proof of Theorem 1.1 Kempe's linkage is used several times as a crucial part of the final mechanical linkage, namely of the addition and multiplication linkage. There we renounce sometimes to the requirement $O, A \in V_{\text {fix }}$ and identify the edge $\{O, A\}$ with an appropriate edge of the addition and multiplication linkage, respectively. In this way we get the possibility of restricting the position of certain vertices orthogonally to $O A$ above the vertex $O$ and without consequence to the considered components of the total configuration space.

Departing from the starting realization $\xi_{0}$ shown in Fig. 4 we obtain other realizations by moving the vertex $X$, such that $X$ lies in a subset of $\operatorname{Im}(\mathbb{C})$, and no $\mathbb{Z}_{2}$-actions are allowed on the vertices of $\mathcal{K}$. First we define the interval $I_{\varepsilon}:=\{z \in \mathbb{C} ; \varepsilon<\operatorname{Im}(z)<$ $d(O, A)-\varepsilon, \operatorname{Re}(z)=0\}$ for any $\varepsilon \in\left[0, \frac{1}{2} d(O, A)\right]$ and obtain the following lemmas:

Lemma 2.9. The vertex $X$ can move on a straight line, namely on $I_{0} \supseteq I_{\varepsilon}$.

Proof. Let the angles $\alpha, \beta, \gamma, \delta, \eta, \rho$ be as shown in Fig. 4. First we consider the edges $\{O, A\},\{O, E\},\{A, B\},\{B, E\}$ of $\mathcal{K}$ : departing from $\xi_{0}$ a motion of $E$ is parameterized by $\rho \in] 0, \pi / 4[$, where $\{A, B\},\{B, E\}$ do not hinder this motion, since $d_{\mathbb{R}}(A, E)<d(A, B)+d(B, E)$. Completing $\mathcal{K}$ by the remaining edges we have $d_{\mathbb{R}}(O, B)<d(O, D)+d(B, D)$ and $d_{\mathbb{R}}(C, E)<d(C, X)+d(E, X)$, so they cause no hindrance for the motion of $E$ too. Second we obtain $X$ at $d(O, E) \cdot e^{i \cdot \rho}+d(E, X) \cdot e^{i(\pi-\delta)}$, where $\delta=\gamma-\eta=((2 \pi-2 \beta-\alpha)-\alpha)-\eta$ and $\eta=\rho=\pi-\alpha-\beta$, i.e., $\delta=\rho$. Since $d(O, E)=d(E, X)$ the vertex $X$ lies at $d(O, E) \cdot\left(e^{i \cdot \rho}-e^{-i \cdot \rho}\right)=2 i \cdot d(O, E) \cdot \sin \rho$, i.e., $X$ moves on $I_{0}$ for $\left.\rho \in\right] 0, \pi / 4[$.

Lemma 2.10. Let $X$ be fixed in $I_{0}$, i.e., $X \in V_{\text {fix }}$ for the moment, such that its position is obtained by a motion departing from $\xi_{0}$ as described in Lemma 2.9. Then a rigid realization $\xi \in[\mathcal{K}]$ results.

Proof. Let $X$ be as prescribed. It follows that $E$ is rigid, since $O \in V_{\text {fix }} ; B$ is also rigid, since $A \in V_{\text {fix }} ; C$ and $D$ are rigid too, because $O \in V_{\text {fix }}$. Therefore the considered realization is rigid.

By the scaling of $I_{\varepsilon}$ we obtain only regular realizations during the motion of $X$, so Lemma 2.6 gives a parameterizing motion of $X$ on $I_{\varepsilon}$. Exactly those realizations are called natural realizations of $\mathcal{K}$.

Lemma 2.11. Let $\varepsilon \in\left[0, \frac{1}{2} d(O, A)\right.$ [. Then an open neighborhood $U_{I_{\varepsilon}}$ in $\mathbb{C}$ of $I_{\varepsilon}$ exists, such that departing from $\xi_{0}$ a motion of $X$ on $U_{I_{\varepsilon}}$ implies a motion of $X$ on $I_{\varepsilon}$.

Proof. By the parameterization of $X$ introduced in the proof of Lemma 2.9 there is $O X \perp O A$, so $X$ lies in $I_{\varepsilon}$. 

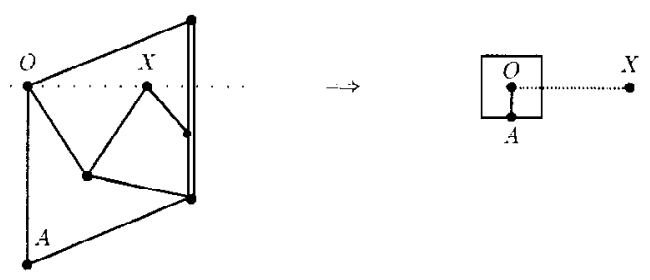

Fig. 5. Simplified representation of Kempe's linkage $\mathcal{K}$.

Summarizing the above we obtain an important corollary, which states intuitively that there exists a one-dimensional part of $[\mathcal{K}]$, which is parameterized by the vertex $X$ in $I_{0}$ and contains the natural realizations of $\mathcal{K}$. In the following $T_{\text {rest }}$ is always any topological space which we do not specify:

Corollary 2.12. For any $\varepsilon \in] 0, \frac{1}{2} d(O, A)\left[\right.$, an open neighborhood $U_{I_{\varepsilon}}$ of $I_{\varepsilon}$ exists such that $\left\{\xi \in[\mathcal{K}] ; X\right.$ in $\left.U_{I_{\varepsilon}}\right\} \approx I_{\varepsilon} \amalg T_{\text {rest }}$, in particular $\left\{\xi \in[\mathcal{K}] ; X\right.$ in $\left.I_{\varepsilon}\right\} \approx I_{\varepsilon} \amalg T_{\text {rest }}$.

All figures in the sections below show Kempe's linkage in a simplified manner, since its operation is determined by the relation between the edge $\{O, A\}$ and the vertex $X$ only. Thus the representation in Fig. 5 enables to change the denotations of the cited vertices $O, A, X$ without any risk of confusion.

\subsection{Product Linkage}

Definition 2.13. Let $\mathcal{G}=\left(V_{\text {fix }} \cup V_{\text {free }}, E, d\right)$ and $\mathcal{G}^{\prime}=\left(V_{\text {fix }}^{\prime} \cup V_{\text {free }}^{\prime}, E^{\prime}, d^{\prime}\right)$ be two mechanical linkages. Then $\mathcal{G} \otimes \mathcal{G}^{\prime}:=\left(V_{\text {fix }} \cup V_{\text {fix }}^{\prime} \cup V_{\text {free }} \cup V_{\text {free }}^{\prime}, E \cup E^{\prime} \cup E_{\text {con }}, d \otimes d^{\prime}\right)$ is called the product linkage of $\mathcal{G}$ and $\mathcal{G}^{\prime}$, where $E_{\text {con }}:=\left\{\left\{V_{1}, V_{1}^{\prime}\right\}\right\}$ with $V_{1} \in V_{\text {fix }}$, $V_{1}^{\prime} \in V_{\text {fix }}^{\prime}$ and $\left.d \otimes d^{\prime}\right|_{E}:=d,\left.d \otimes d^{\prime}\right|_{E^{\prime}}:=d^{\prime}, d \otimes d^{\prime}\left(V_{1}, V_{1}^{\prime}\right):=d_{\mathbb{R}}\left(V_{1}, V_{1}^{\prime}\right)$.

Lemma 2.14. $\left[\mathcal{G} \otimes \mathcal{G}^{\prime}\right] \approx[\mathcal{G}] \times\left[\mathcal{G}^{\prime}\right]$

Proof. $\mathcal{G}$ and $\mathcal{G}^{\prime}$ independently create the configuration spaces $[\mathcal{G}]$ and $\left[\mathcal{G}^{\prime}\right]$.

\section{Proof of Theorem 1.1}

1. We are interested in compact real algebraic varieties in $\mathbb{R}^{q}$ that occur as the zero set of a single polynomial $F \in \mathbb{R}\left[X_{1}, \ldots, X_{q}\right]$.

Consider the canonical representation of $F \in \mathbb{R}\left[X_{1}, \ldots, X_{q}\right]$ as a finite real linear combination of the monomials $X_{1}^{v_{1}} \cdots X_{q}^{v_{q}} \in \mathbb{R}\left[X_{1}, \ldots, X_{q}\right]$ :

$$
F=\sum_{\left(v_{1}, \ldots, v_{q}\right) \in \mathbb{N}_{0}^{q}} a_{v_{1}, \ldots, v_{q}} X_{1}^{v_{1}} \cdots X_{q}^{v_{q}}
$$

with $a_{v_{1}, \ldots, v_{q}} \in \mathbb{R}$, all but a finite number are zero. 
The polynomial $F$ is constructed algorithmically from the zero polynomial with addition and multiplication of the terms $a_{v_{1}, \ldots, v_{q}}$ and $X_{1}, \ldots, X_{q}$. First we define the lexicographic order $\geq$ on $\mathbb{N}_{0}^{q}$ and get $\left(\mathbb{N}_{0}^{q}, \geq\right)$ :

Definition 3.1. Let $\left(v_{1}, \ldots, v_{q}\right)$ and $\left(v_{1}^{\prime}, \ldots, v_{q}^{\prime}\right)$ be in $\mathbb{N}_{0}^{q}$. Then $\left(v_{1}, \ldots, v_{q}\right) \geq$ $\left(v_{1}^{\prime}, \ldots, v_{q}^{\prime}\right)$ if and only if $v_{1}>v_{1}^{\prime}$ or $\left(v_{1}=v_{1}^{\prime}\right.$ and $\left.v_{2}>v_{2}^{\prime}\right)$ or $\ldots$ or $\left(v_{1}=v_{1}^{\prime}, \ldots\right.$, $\left.v_{q}=v_{q}^{\prime}\right)$.

Constructing $F$ we start with the smallest element $(0, \ldots, 0) \in \mathbb{N}_{0}^{q}$ and define the set $\mathcal{U}_{\mathrm{s}}(F)$ of special subpolynomials of $F$, consisting of the following elements:

$$
u_{(0, \ldots, 0)}^{(0, \ldots, 0)}:=a_{0, \ldots, 0} \quad \text { and } \quad u_{(1,0, \ldots, 0)}^{(0, \ldots, 0)}:=a_{1,0, \ldots, 0}, \quad u_{(1,0, \ldots, 0)}^{(1,0, \ldots, 0)}:=a_{1,0, \ldots, 0} X_{1} .
$$

The construction proceeds in this manner up to the biggest multi-index $\left(\hat{v}_{1}, \ldots, \hat{v}_{q}\right)$ that appears in $F$. As a result we get the monomial of maximal total degree multiplied by its coefficient in $F$ :

$$
\begin{array}{lll}
u_{\left(\hat{v}_{1}, \ldots, \hat{v}_{q}\right)}^{(0, \ldots, 0)}:=a_{\hat{v}_{1}, \ldots, \hat{v}_{q}} & \cdots & u_{\left(\hat{v}_{1}, \ldots, \hat{v}_{q}\right)}^{\left(\iota_{1}, \ldots, l_{1}\right)}:=a_{\hat{v}_{1}, \ldots, \hat{v}_{q}} X_{1}^{l_{1}} \cdots X_{n}^{l_{q}} \\
& \cdots & u_{\left(\hat{v}_{1}, \ldots, \hat{v}_{q}\right)}^{\left(\hat{v}_{1}\right)}:=a_{\hat{v}_{1}, \ldots, \hat{v}_{q}} X_{1}^{\hat{v}_{1}} \cdots X_{n}^{\hat{v}_{q}} .
\end{array}
$$

Further we define

$$
\mathcal{U}_{\mathrm{c}}(F):=\left\{v^{\left(v_{1}, \ldots, v_{q}\right)}:=\sum_{\left(\iota_{1}, \ldots, l_{q}\right) \leq\left(v_{1}, \ldots, v_{q}\right)} u_{\left(\iota_{1}, \ldots, l_{q}\right)}^{\left(\iota_{1}, \ldots, l_{q}\right)} ;\left(v_{1}, \ldots, v_{q}\right) \leq\left(\hat{v}_{1}, \ldots, \hat{v}_{q}\right)\right\}
$$

as the set of composed subpolynomials of $F$ and the union of all special and composed subpolynomials $\mathcal{U}(F):=\mathcal{U}_{\mathrm{s}}(F) \cup \mathcal{U}_{\mathrm{c}}(F)$.

Example 3.2. Consider the polynomial $F(X, Y):=5 X^{2}+3 Y^{2}-2$ in two variables. Then $\mathcal{U}_{\mathrm{s}}(F)=\left\{-2,5,5 X, 5 X^{2}, 3,3 Y, 3 Y^{2}\right\}$ and $\mathcal{U}_{\mathrm{c}}(F)=\left\{-2,-2+5 X^{2},-2+5 X^{2}+\right.$ $\left.3 Y^{2}\right\}$.

Since $V$ is compact there exists $r \in \mathbb{R}_{+}^{*}$, such that $V \subseteq B_{r}(0):=\left\{x \in \mathbb{R}^{q} ; x \leq\right.$ $r\}$. Define the real numbers $A_{F}:=\max \left\{|u(x)| ; \forall x \in B_{r+1}(0), \forall u \in \mathcal{U}(F)\right\}, B_{F}:=$ $\max \left\{\left|x_{j}\right| ; \forall x \in B_{r+1}(0), \forall j \in\{1, \ldots, q\}\right\}=r+1$, and

$$
L_{F}:=1+\max \left\{A_{F}, B_{F}\right\} .
$$

Since $\mathcal{U}(F) \subset \mathbb{R}\left[X_{1}, \ldots, X_{q}\right]$ and $B_{r+1}(0)$ is compact, these extrema exist.

2. With the product linkage $\mathcal{K}_{1} \otimes \cdots \otimes \mathcal{K}_{q}$ of $q$ identical linkages of Kempe a system of coordinates on $]-L_{F}, L_{F}\left[{ }^{q}\right.$ is constructed, such that each vertex $X_{1}, \ldots, X_{q}$ represents a coordinate.

For this purpose we set for all $j \in\{1, \ldots, q\}$ the length $d\left(O_{j}, A_{j}\right):=2 L_{F}+2$ in $\mathcal{K}_{j}$. Define $V_{\text {fix }}$ of $\mathcal{K}_{1} \otimes \cdots \otimes \mathcal{K}_{q}$ as the set $\left\{O_{1}, A_{1}, \ldots, O_{q}, A_{q}\right\}$, such that all $O_{j}$ are pinned 
down at $\left(-L_{F}-1,0\right)$ and all $A_{j}$ at $\left(-L_{F}-1,-2 L_{F}-2\right)$ in $\mathbb{R}^{2}$. Then each vertex $X_{j}$ can be moved, parameterizing in the subset

$$
\left.I_{F}:=\right]-L_{F}, L_{F}[\times\{0\} .
$$

By Lemma 2.14 we obtain $\left[\mathcal{K}_{1} \otimes \cdots \otimes \mathcal{K}_{q}\right]=\left[\mathcal{K}_{1}\right] \times \cdots \times\left[\mathcal{K}_{q}\right]$ and by Corollary 2.12 the restriction of the vertices $X_{1}, \ldots, X_{q}$ in $I_{F}$ is

$$
\begin{aligned}
\left\{\xi \in \left[\mathcal{K}_{1}\right.\right. & \left.\left.\otimes \cdots \otimes \mathcal{K}_{q}\right] ;\left(X_{1}, \ldots, X_{q}\right) \text { in }\left(I_{F}\right)^{q}\right\} \\
& \left.:=\left\{\xi \in\left[\mathcal{K}_{1} \otimes \cdots \otimes \mathcal{K}_{q}\right] ; X_{1}, \ldots, X_{q} \text { in } I_{F}\right\} \approx\right]-L_{F}, L_{F}\left[{ }^{q} \amalg T_{\text {rest }} .\right.
\end{aligned}
$$

Note that the realizations of $\mathcal{K}_{1} \otimes \cdots \otimes \mathcal{K}_{q}$ are called natural, if for all $j \in\{1, \ldots, q\}$ the realizations of $\mathcal{K}_{j}$ are natural. Our next aim is to couple the special mechanical linkages in a way that they define addition and multiplication on $I_{F}$.

3. Required properties for the addition linkage $\mathcal{A}$ and the multiplication linkage $\mathcal{M}$.

Let $x$ and $y$ be the evaluations of two subpolynomials $u, v \in \mathcal{U}(F)$ at the point $x_{0} \in$ $B_{r+1}(0)$, i.e., $u\left(x_{0}\right)=x$ and $v\left(x_{0}\right)=y$. If $u+v, u \cdot v, a+u$, or $a \cdot u$ are elements of $\mathcal{U}(F)$, then using construction in part 1 we assume that $a, x, y \in]-L_{F}, L_{F}[$ and $x+y$, $x \cdot y, a+x$, or $a \cdot x \in]-L_{F}, L_{F}$, where $a$ is any coefficient of $F$. For each mechanical linkage $\mathcal{A}$ and $\mathcal{M}$, the values of $x$ and $y$ as well as the evaluations $x+y$ and $x \cdot y$ are attached to the corresponding vertices $X, Y, X+Y, X \cdot Y$ in $I_{F}$. Then we need to have the following properties:

(P1) The mechanical linkages $\mathcal{A}$ and $\mathcal{M}$ can be placed in such a way that $X$ and $Y$ move on $I_{F}$.

(P2) If both $X, Y$ of $\mathcal{A}$ are fixed in $I_{F}$, then a rigid realization $\xi \in[\mathcal{A}]$ results. If both $X, Y$ of $\mathcal{M}$ are fixed in $I_{F}$, then a rigid realization $\xi \in[\mathcal{M}]$ results.

(P3) All realizations obtained in (P1) are regular.

(P4) The construction of the addition and multiplication with $\mathcal{A}$ and $\mathcal{M}$ on $I_{F}$ is well defined in the sense that for each realization obtained in (P2) the evaluation vertices $X+Y$ of $\mathcal{A}$ and $X \cdot Y$ of $\mathcal{M}$ represent the evaluations $x+y$ and $x \cdot y$ in $I_{F}$.

Conditions (P1)-(P3) ensure by Lemma 2.6, that the used motion of $X, Y$ is parameterizing for both mechanical linkages $\mathcal{A}$ and $\mathcal{M}$.

\section{Addition linkage $\mathcal{A}$.}

The mechanical linkage $\mathcal{A}$ which is responsible for the addition consists of two translation linkages $\mathcal{T}_{1}, \mathcal{T}_{2}$, two linkages of Kempe $\mathcal{K}_{1}, \mathcal{K}_{2}$, and two edges $E_{1}, E_{2}$ as shown in Fig. 6.

We take the following attachments and sizes, where $c>1$ is any real constant for the moment:

(a) The set of vertices of $\mathcal{T}_{1}$ is $\{A, B, C, D, G, H\}$ such that $d(A, B)=d(A, G)=$ $d(G, H)=2 c \cdot L_{F}$. 


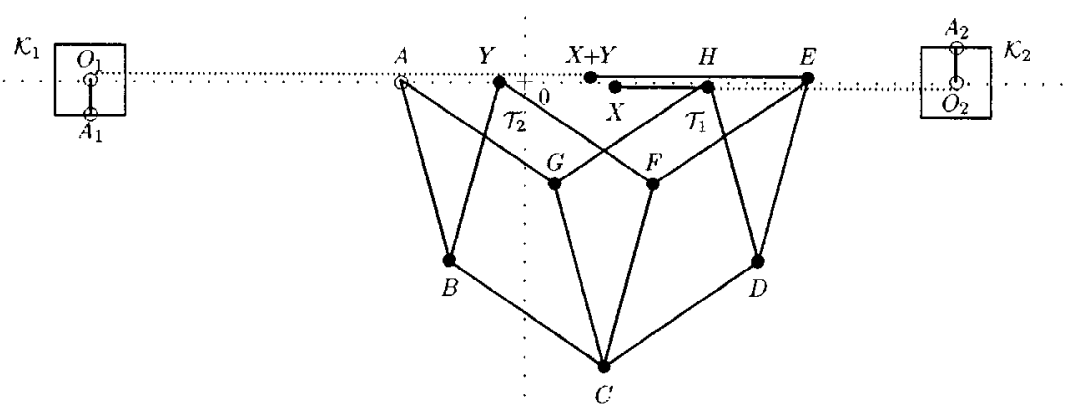

Fig. 6. Starting realization $\xi_{0}$ of the addition linkage $\mathcal{A}$.

(b) The set of vertices of $\mathcal{T}_{2}$ is $\{B, C, D, E, F, Y\}$ such that $d(B, Y)=d(F, Y)=$ $d(E, F)=2 c \cdot L_{F}$.

(c) Each $\mathcal{K}_{j}$ for $j \in\{1,2\}$ is given by $d\left(O_{j}, A_{j}\right)=8 c \cdot L_{F}$.

(d) The edge $E_{1}$ is given by $\{E, X+Y\}$ such that $d(E, X+Y)=3 c \cdot L_{F}$, and $E_{2}$ is given by $\{H, X\}$ such that $d(H, X)=c \cdot L_{F}$.

To have the properties of part 3 the set $V_{\text {fix }}$ of $\mathcal{A}$ is $\left\{O_{1}, A_{1}, O_{2}, A_{2}, A\right\}$, such that $O_{1}$ is pinned down at $\left(-4 c \cdot L_{F}, 0\right), A_{1}$ at $\left(-4 c \cdot L_{F},-8 c \cdot L_{F}\right), O_{2}$ at $\left(4 c \cdot L_{F}, 0\right), A_{2}$ at $\left(4 c \cdot L_{F}, 8 c \cdot L_{F}\right)$, and $A$ at $\left(-2 c \cdot L_{F}, 0\right)$. The mechanical linkage $\mathcal{A}_{a}$ for the addition with a constant $a \in]-L_{F}, L_{F}$ [ is obtained from $\mathcal{A}$ by completing $V_{\text {fix }}$ with the vertex $Y$ pinned down at $(a, 0)$.

Before verifying properties (P1)-(P4) we give a description of the working method of $\mathcal{A}$. Let $X, Y$ be in $I_{F}$. The vertex $E$ is gained by a shift of the equilateral triangle $A B Y$ to $H D E$ using both translation linkages $\mathcal{T}_{1}$ and $\mathcal{T}_{2}$. Finally to get $X+Y$ we subtract the vector $(0-A)+(H-X)$ from $E$, whose length is $3 c \cdot L_{F}$. Note that this subtraction is necessary, since $H$ is displaced from $X$ by a length of $c \cdot L_{F}$ and $A$ is pinned down at $\left(-2 c \cdot L_{F}, 0\right)$, i.e., is not equal to the origin of $I_{F}$. The position of $X+Y$ and $H$ are forced to $\mathbb{R} \times\{0\}$ by $\mathcal{K}_{1}$ and $\mathcal{K}_{2}$, respectively. Both translations along $\mathbb{R} \times\{0\}$ ensure (P2) and (P3) for any $X, Y$ on $I_{F}$ if $c \gg 1$.

Verification of $(P 1)-(P 4)$ for $\mathcal{A}$.

(P1) We consider the starting realization $\xi_{0}$ shown in Fig. 6 . If $X$ is fixed in $I_{F}$, then $\mathcal{K}_{2}$ ensures by Lemma 2.11 that the vertex $H$ is rigid, so $G$ is determined modulo $\mathbb{Z}_{2}$-actions which are not of interest. Under this condition both $\mathcal{T}_{1}$ and $\mathcal{T}_{2}$ allow realizations of $\mathcal{A}$ such that we have congruent, nondegenerate triangles $A B Y$, $G C F$, and $H D E$ : they may be obtained by moving $Y$ on $I_{F}$. Note that $\mathcal{K}_{1}, \mathcal{K}_{2}$, $E_{1}$, and $E_{2}$ are without significance for this motion of $Y$ : for any $X$ in $I_{F}$ the scaling of $\mathcal{K}_{1}, E_{1}, E_{2}$ ensures that they do not hinder the motion of $Y$ up to the range of $I_{F}$. By symmetry we obtain the same result for a motion of $X$ while $Y$ is fixed: the scaling of $\mathcal{K}_{2}, E_{1}, E_{2}$ ensures that they do not hinder the motion of $X$ up to the range of $I_{F}$. Summarizing we get departing from $\xi_{0}$ a motion of $X, Y$ on $I_{F}$. 
(P2) First let $\xi \in[\mathcal{A}]$ be any realization obtained in (P1), such that $X, Y$ are fixed in $I_{F}$. Then $H$ is rigid and so $B$ and $G$ are rigid. Thus $C$ is rigid since $B$ does not lie at the same point as $G$. It follows that $F$ and $D$ are rigid and therefore $E$ is rigid too. By Lemma 2.10 the linkages of Kempe $\mathcal{K}_{1}$ and $\mathcal{K}_{2}$ are rigid, which implies (P2) for any realization of $\mathcal{A}$ described in (P1).

(P3) Choose $c \gg 1$. Then departing from $\xi_{0}$ a motion of $X, Y$ on $I_{F}$ induces only small deformations of $\mathcal{T}_{1}, \mathcal{T}_{2}, \mathcal{K}_{1}$, and $\mathcal{K}_{2}$, especially the triangles of vertices containing only two edges never degenerate. Thus all realizations of $\mathcal{A}$ obtained above are regular and only those are then called natural realizations of $\mathcal{A}$.

(P4) This property follows by (P1) and by (P2), i.e., the rigidity of $\mathcal{A}$, whenever $X, Y$ are fixed in $I_{F}$, where we always take into consideration the described working method of $\mathcal{A}$.

\section{Multiplication linkage $\mathcal{M}$.}

The description of the multiplication with a mechanical linkage $\mathcal{M}$ is based on the following modification of a well-known construction with a pair of compasses and a ruler suggested in Fig. 7. For any real $\lambda \geq 1$ the cutting point of the lines $\mathbb{R} \times\{0\}$ and $g_{y}$ is $x \cdot \lambda \quad y:=\left(\lambda^{-1} x \cdot y, 0\right)$, where $g_{y}$ goes through $y^{\prime}:=(0, y)$ and is parallel to $g_{x}$ given by the points $(0, \lambda)$ and $x:=(x, 0)$. It is easy to see that this construction defines the multiplication of $x, y$ and $\lambda^{-1}$ by the ratio $x: \lambda=\left(\lambda^{-1} x y\right): y$, whenever $y:=(y, 0) \neq 0$. If $y=0$, then $g_{y}$ cuts $\mathbb{R} \times\{0\}$ in 0 for any $x \in \mathbb{R} \times\{0\}$, thus $x \cdot \lambda \quad y=0$ as prescribed.

We focus our attention on the first part of the multiplication linkage $\mathcal{M}$, which is responsible for the rotation of the vertex $Y$ anticlockwise around the origin by $\pi / 2$ to $Y^{\prime}$, whenever $Y$ lies in $I_{F}$. The corresponding mechanical linkage $\mathcal{M}_{C}$ is a coupling of a conformal linkage $\mathcal{C}$ and two translation linkages $\mathcal{T}_{1}$ and $\mathcal{T}_{2}$ as Fig. 8 shows.

We take the following attachments and sizes:

(a) The set of vertices of $\mathcal{C}$ is given by the denotations in Section 2.3.2 such that $d(O, B)=d\left(O, B^{\prime}\right)=L_{F}$ and $d\left(A, A^{\prime}\right)=(\sqrt{2} / 2) L_{F}$.

(b) The set of vertices of $\mathcal{T}_{1}$ is $\left\{O, O_{0}, C, D, F, Y\right\}$ such that $d\left(O, O_{0}\right)=d(O, D)=$ $d(C, D)=L_{F}$.

(c) The set of vertices of $\mathcal{T}_{2}$ is $\left\{O, O_{0}^{\prime}, B^{\prime}, C^{\prime}, F^{\prime}, Y^{\prime}\right\}$ such that $d\left(O, O_{0}^{\prime}\right)=$ $d\left(O, B^{\prime}\right)=d\left(B^{\prime}, C^{\prime}\right)=L_{F}$.

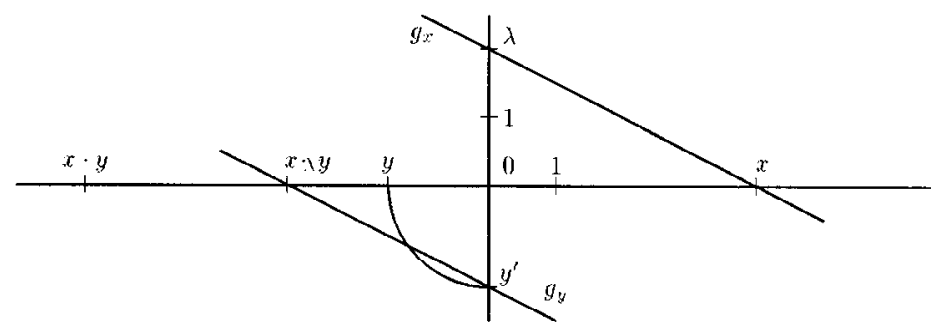

Fig. 7. Construction of $x \cdot y$. 


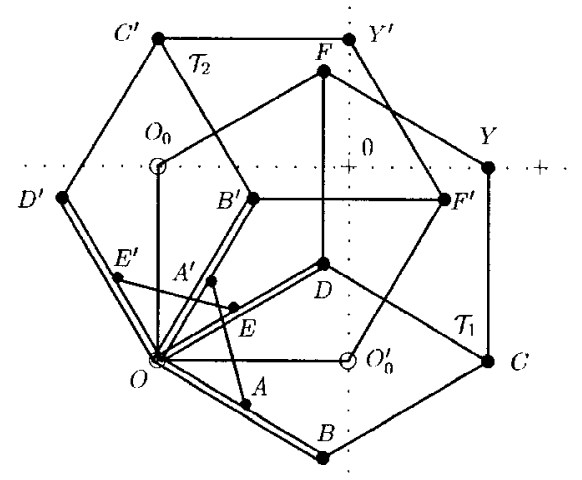

Fig. 8. Starting realization $\xi_{0}$ of the part $\mathcal{M}_{C}$ of the multiplication linkage $\mathcal{M}$.

In order to have the properties of part 3, there is $\left\{O, O_{0}, O_{0}^{\prime}\right\} \subset V_{\text {fix }}$, such that $O$ is pinned down at $\left(-L_{F},-L_{F}\right), O_{0}$ at $\left(-L_{F}, 0\right)$ and $O_{0}^{\prime}$ at $\left(0,-L_{F}\right)$, where $V_{\text {fix }}$ is already related to $\mathcal{M}$.

Lemma 3.3. Departing from the starting realization $\xi_{0}$ of $\mathcal{M}_{C}$, the vertex $Y$ can be moved parameterizing on $I_{F}$, and for $Y$ at any point $(y, 0) \in I_{F}$ there is $Y^{\prime}$ at the point $(0, y)$.

Proof. Consider the starting realization $\xi_{0}$ and $\mathcal{C}$ in Fig. 8. A motion of $C$ on ]$-L_{F}, L_{F}\left[\times\left\{-L_{F}\right\}\right.$ produces a similar motion of $C^{\prime}$ on $\left.\left\{-L_{F}\right\} \times\right]-L_{F}, L_{F}[$, since both kite quadrilaterals $O B C D$ and $O B^{\prime} C^{\prime} D^{\prime}$ are forced to be congruent by the edges $\left\{A, A^{\prime}\right\}$ and $\left\{E, E^{\prime}\right\}$. The prescribed coupling of $\mathcal{T}_{1}$ and $\mathcal{T}_{2}$ causes a vertical shift of $C$ to $Y$ in $I_{F}$ and a horizontal shift of $C^{\prime}$ to $Y^{\prime}$ in $\left.\{0\} \times\right]-L_{F}, L_{F}[$, in particular no hindrance for this motion.

We prove that $\mathcal{M}_{C}$ is rigid and fix $Y$ after a motion at any point in $I_{F}$ as above. Then $\mathcal{T}_{1}$ is determined modulo $\mathbb{Z}_{2}$-actions which are not of interest, i.e., $\mathcal{T}_{1}$ is rigid. Thus $C$ is rigid and since $O \in V_{\text {fix }}$ with $O$ and $C$ at different points, $\mathcal{C}$ is rigid too. Finally $\mathcal{T}_{2}$ is rigid, since $C^{\prime}$ and $O$ do not lie at the same point.

Every realization of $\mathcal{M}_{C}$ is regular during the motion of $Y$ on $I_{F}$, since $Y$ never lies at the point $\left(-L_{F}, 0\right)$ and so both translation linkages do not degenerate.

Finally with Lemma 2.6 a parameterizing motion of $Y$ on $I_{F}$ results and by symmetry of the construction there is $d_{\mathbb{R}}\left(0, Y^{\prime}\right)=d_{\mathbb{R}}(0, Y)$.

The last part of $\mathcal{M}$ has to define the linear transformation of the vertex $X \cdot \lambda Y$ in $\left.\lambda^{-1} I_{F}:=\right]-\lambda^{-1} L_{F}, \lambda^{-1} L_{F}\left[\times\{0\}\right.$ to the evaluation vertex $X \cdot Y$ in $I_{F}$. The corresponding mechanical linkage $\mathcal{M}_{L}$ is a coupling of a linear linkage $\mathcal{L}$ and a linkage of Kempe $\mathcal{K}_{4}$ as in Fig. 9.

We take the following attachments and sizes, where $\lambda:=2 c \cdot L_{F}$ is already related 


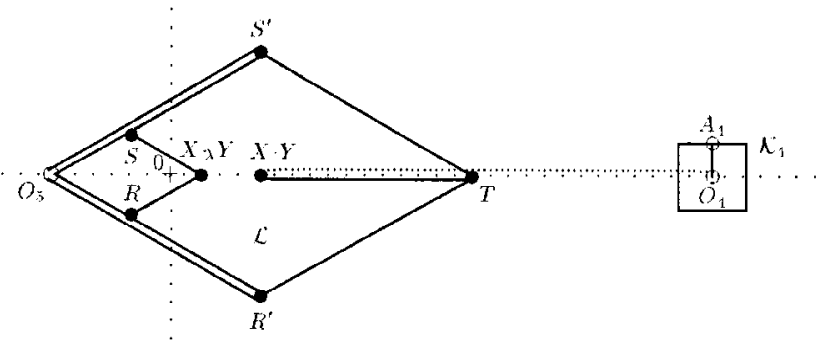

Fig. 9. Starting realization $\xi_{0}$ of the part $\mathcal{M}_{L}$ of the multiplication linkage $\mathcal{M}$.

to $\mathcal{M}$, and $c>1$ is any real constant for the moment:

(j) The set of vertices of $\mathcal{L}$ is $\left\{O_{5}, R, R^{\prime}, S, S^{\prime}, T, X \cdot \lambda Y\right\}$ such that $d\left(O_{5}, R\right)=$ $1 / c$ and $d\left(O_{5}, R^{\prime}\right)=\lambda / c=2 L_{F}$.

(k) $\mathcal{K}_{4}$ is given by $d\left(O_{4}, A_{4}\right)=4 L_{F}$.

(l) The edge $E_{3}$ is given by $\{T, X \cdot Y\}$ such that $d(T, X \cdot Y)=2 L_{F}-1 / c$.

In order to have the properties of part 3, there is $\left\{O_{4}, O_{5}, A_{4}\right\} \subset V_{\text {fix }}$, such that $O_{4}$ is pinned down at $\left(2 L_{F}, 0\right), O_{5}$ at $(-1 / c, 0)$, and $A_{4}$ at $\left(2 L_{F}, 4 L_{F}\right)$, where $V_{\text {fix }}$ is already related to $\mathcal{M}$. Notice that for $\lambda=2 c \cdot L_{F}$ there is $\left.\lambda^{-1} I_{F}=\right]-1 / 2 c, 1 / 2 c[\times\{0\}$.

Lemma 3.4. Let $c>1$ be any real constant and $\lambda=2 c \cdot L_{F}$. Departing from the starting realization $\xi_{0}$ of $\mathcal{M}_{L}$, the vertex $X \cdot \lambda Y$ can be moved parameterizing on $\lambda^{-1} I_{F}$, and for $X \cdot \lambda Y$ at any point $\left(\lambda^{-1} x \cdot y, 0\right) \in \lambda^{-1} I_{F}$ there is $X \cdot Y$ at the point $(x \cdot y, 0) \in I_{F}$.

Proof. Consider first $\mathcal{L}$ in Fig. 9 showing the starting realization $\xi_{0}$ of $\mathcal{M}_{L}$. Clearly, $X \cdot \lambda Y$ can be moved on $\lambda^{-1} I_{F}$, since there is $\left.d_{\mathbb{R}}\left(0_{5}, X \cdot \lambda Y\right) \in\right] 1 / 2 c, 3 / 2 c[$, thus $d_{\mathbb{R}}\left(0_{5}, X \cdot \lambda Y\right)<2 / c=d\left(O_{5}, R\right)+d(R, X \cdot \lambda Y)$. By its scaling, $\mathcal{K}_{4}$ does not hinder the induced motion of $T$ and $X \cdot Y$, because it allows $X \cdot Y$ in $I_{F}$.

By fixing $X \cdot \lambda Y$ at any point during the motion above, there are $R, S$ rigid, since $X \cdot \lambda Y$ never lies at $(-1 / c, 0)$. Thus $R^{\prime}, S^{\prime}$, and $T$ are rigid. Kempe's linkage $\mathcal{K}_{4}$ ensures by Lemma 2.11 that $X \cdot Y$ lies on $\mathbb{R} \times\{0\}$, so $X \cdot Y$ is rigid and $\mathcal{K}_{4}$ too because of Lemma 2.10.

During the motion of $X \cdot \lambda Y$ we obtain only regular realizations of $\mathcal{L}$ and $\mathcal{K}_{4}$. Because of their connection by $E_{3}, \mathcal{M}_{L}$ is regular too.

Summarizing by Lemma 2.6 the motion of $X \cdot_{\lambda} Y$ on $\lambda^{-1} I_{F}$ is parameterizing, where $X \cdot Y$ lies on $(z, 0)$ with $z=\lambda \cdot x \cdot \lambda y+(1 / c)(\lambda-1)-d(X \cdot Y, T)=\lambda \cdot x \cdot \lambda y$.

Figure 10 shows the multiplication linkage $\mathcal{M}$, where $\mathcal{M}_{C}$ and $\mathcal{M}_{L}$ are indicated only by their vertices $Y^{\prime}$ and $X \cdot_{\lambda} Y$, respectively, since their operation is determined by Lemmas 3.3 and 3.4. The multiplication linkage $\mathcal{M}$ is completed by three translation linkages $\mathcal{T}_{3}, \mathcal{T}_{4}, \mathcal{T}_{5}$, three linkages of Kempe $\mathcal{K}_{1}, \mathcal{K}_{2}, \mathcal{K}_{3}$, and two edges $E_{1}, E_{2}$ with the 


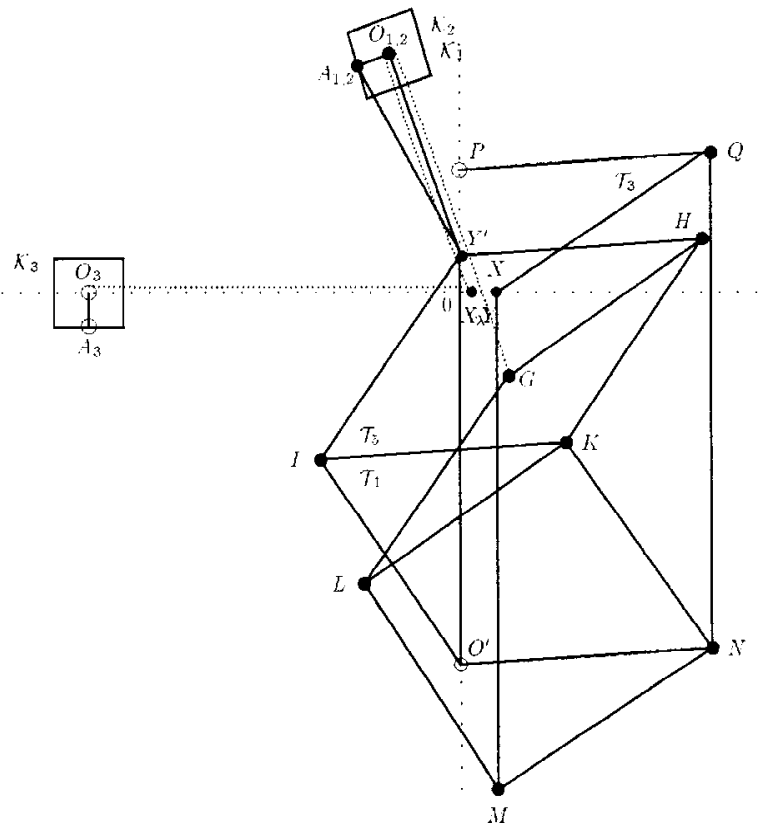

Fig. 10. Starting realization $\xi_{0}$ of the multiplication linkage $\mathcal{M}$.

following attachments and sizes, where $c>1$ is as for $\mathcal{M}_{L}$ :

(d) The set of vertices of $\mathcal{T}_{3}$ is $\left\{O^{\prime}, M, N, P, Q, X\right\}$ such that $d\left(O^{\prime}, N\right)=$ $d(M, N)=4 c \cdot L_{F}$ and $d\left(O^{\prime}, P\right)=8 c \cdot L_{F}$.

(e) The set of vertices of $\mathcal{T}_{4}$ is $\left\{O^{\prime}, I, K, L, M, N\right\}$ such that $d\left(O^{\prime}, N\right)=d(M, N)=$ $d\left(O^{\prime}, I\right)=4 c \cdot L_{F}$.

(f) The set of vertices of $\mathcal{T}_{5}$ is $\left\{G, H, I, K, L, Y^{\prime}\right\}$ such that $d(I, K)=d(K, L)=$ $d(G, L)=4 c \cdot L_{F}$.

(g) $\mathcal{K}_{1}$ and $\mathcal{K}_{2}$ are given by $d\left(O_{1,2}, A_{1,2}\right)=8 c \cdot L_{F}$.

(h) $\mathcal{K}_{3}$ is given by $d\left(O_{3}, A_{3}\right)=8 c \cdot L_{F}$.

(i) The edge $E_{1}$ is given by $\left\{O_{1,2}, Y^{\prime}\right\}$ such that $d\left(O_{1,2}, Y^{\prime}\right)=4 c \cdot L_{F}$, and $E_{2}$ is given by $\left\{A_{1,2}, Y^{\prime}\right\}$ such that $d\left(A_{1,2}, Y^{\prime}\right)=4 \sqrt{5} c \cdot L_{F}$.

To have the properties of part 3 the set $V_{\text {fix }}$ of $\mathcal{M}$ is $\left\{O, O^{\prime}, O_{0}, O_{0}^{\prime}, O_{3}, O_{4}, O_{5}, A_{3}\right.$, $\left.A_{4}, P\right\}$, such that $O, O_{0}, O_{0}^{\prime}, O_{4}, O_{5}, A_{4}$ are pinned down as prescribed for $\mathcal{M}_{C}$ and $\mathcal{M}_{L}, O^{\prime}$ at $\left(0,-6 c \cdot L_{F}\right), O_{3}$ at $\left(-6 c \cdot L_{F}, 0\right), A_{3}$ at $\left(-6 c \cdot L_{F},-8 c \cdot L_{F}\right)$, and $P$ at $\left(0,2 c \cdot L_{F}\right)$, i.e., $d_{\mathbb{R}}(0, P)=\lambda$ as in Lemma 3.4. The mechanical linkage $\mathcal{M}_{a}$ for the multiplication with a constant $a \in]-L_{F}, L_{F}$ [ is obtained from $\mathcal{M}$ by completing $V_{\text {fix }}$ with the vertex $Y$ pinned down at $(a, 0)$.

Before verifying properties $(\mathrm{P} 1)-(\mathrm{P} 4)$ we give a description of $\mathcal{M}$ related to the geometrical principle of the multiplication explained above. Let $X, Y$ be in $I_{F}$ such that $x \cdot y \in]-L_{F}, L_{F}$ [. Then $g_{x}$ is given by $P, X$, and by the vertices $O^{\prime}, M$ of the translation linkage $\mathcal{T}_{3}$ a line parallel to $g_{x}$ is defined. By $\mathcal{T}_{4}, \mathcal{T}_{5}$ the line $g_{y}$ through $G, Y^{\prime}$ is defined, 
where $Y^{\prime}$ is obtained by $\mathcal{M}_{C}$ as a rotation of $Y$ described above. Kempe's linkage $\mathcal{K}_{1}$ hands over the direction of $g_{y}$ to $\mathcal{K}_{2}$ in a way, such that $O_{1,2} A_{1,2}$ is orthogonal to $g_{y}$. We obtain $x \cdot \lambda \quad y$ as the common vertex $X \cdot \lambda Y$ of $\mathcal{K}_{2}$ and $\mathcal{K}_{3}$, which is the cutting point between $g_{y}$ and $\mathbb{R} \times\{0\}$. Finally $\mathcal{M}_{L}$ defines the evaluation vertex $X \cdot Y$ as the linear transformation of $X \cdot \lambda Y$ on $\lambda^{-1} I_{F}$ by multiplication with $\lambda$. By $d_{\mathbb{R}}(0, P)=\lambda>L_{F}$ we avoid a motion of $Y^{\prime}$ through $P$, which simplifies considerably the proof of (P3) using only small deformations of the multiplication linkage departing from $\xi_{0}$.

Verification of $(P 1)-(P 4)$ for $\mathcal{M}$.

(P1) We consider the starting realization $\xi_{0}$ of $\mathcal{M}$, and to begin with we disregard $\mathcal{T}_{4}, \mathcal{T}_{5}, \mathcal{K}_{1}, \mathcal{K}_{2}, \mathcal{K}_{3}, E_{1}, E_{2}, \mathcal{M}_{L}$. It is clear that $\mathcal{T}_{3}$ allows a motion of the vertex $X$ in $I_{F}$, since $d_{\mathbb{R}}(P, X)<8 c \cdot L_{F}$ is always fulfilled and by $\mathcal{M}_{C}$ the vertex $Y$ can be moved on any point $(y, 0)$ in $I_{F}$ and induces by Lemma 3.3 a position of $Y^{\prime}$ at $(0, y)$. Both translation linkages $\mathcal{T}_{4}, \mathcal{T}_{5}$ do not hinder the motion of $X$, since $X$ determines only the triangles $O^{\prime} M N, I K L$ and $G H Y^{\prime}$ of $\mathcal{T}_{4}, \mathcal{T}_{5}$, which are obtained by shifting $P Q X$ of $\mathcal{T}_{3}$. Neither are they hindering the motion of $Y$, since $d_{\mathbb{R}}\left(O^{\prime}, Y^{\prime}\right)<8 c \cdot L_{F}$. By their scaling the mechanical linkages $\mathcal{K}_{1}$, $\mathcal{K}_{2}, \mathcal{K}_{3}, E_{1}, E_{2}, \mathcal{M}_{L}$ are without importance for the motions of $X, Y$ on $I_{F}$, whenever $x \cdot y \in$ ] $-L_{F}, L_{F}$ [, i.e., $X \cdot \lambda Y$ lies in $\lambda^{-1} I_{F}$. Summarizing we get departing from $\xi_{0}$ a motion of $(X, Y)$ on $\left\{(x, y) \in(]-L_{F}, L_{F}[\times\{0\})^{2} ; x \cdot y \in\right.$ ]$-L_{F}, L_{F}[\} \subset I_{F}^{2}$.

(P2) Let $\xi \in[\mathcal{M}]$ be any realization obtained in (P1), such that $X, Y$ are fixed in $I_{F}$. Then $\mathcal{T}_{3}$ is rigid, since $P$ does not lie at the same point as $X$ and $\mathcal{M}_{C}$ is rigid by Lemma 3.3. Therefore both $\mathcal{T}_{4}, \mathcal{T}_{5}$ are rigid, since $Y^{\prime}$ and $O^{\prime}$ are at different points. Lemmas 2.10 and 2.11 ensure that the linkages of Kempe $\mathcal{K}_{1}, \mathcal{K}_{2}, \mathcal{K}_{3}$ are all rigid. So by Lemma 3.4 $\mathcal{M}_{L}$ is rigid which implies (P2).

(P3) Choose $c \gg 1$. Then departing from $\xi_{0}$ a motion of $(X, Y)$ as in (P1) induces only small deformations of $\mathcal{T}_{3}, \mathcal{T}_{4}, \mathcal{T}_{5}, \mathcal{K}_{1}, \mathcal{K}_{2}$, and $\mathcal{K}_{3}$, especially the triangles of vertices consisting of two edges never degenerate. By connecting the vertex $Y^{\prime}$ of $\mathcal{M}_{C}$ and $X \cdot_{\lambda} Y$ of $\mathcal{M}_{L}$ to the linkages cited above, condition (ii) of Lemma 2.6 may be injured. This is unimportant for the claimed parameterizing motion of $X, Y$, since the motion of $Y$ defines the parameterization of $Y^{\prime}$, and the motion of $X, Y^{\prime}$ gives $X \cdot \lambda \quad Y$ and so $X \cdot Y$. Thus all realizations of $\mathcal{M}$ obtained above are called natural realizations of $\mathcal{M}$.

(P4) This property follows by (P1) and (P2), i.e., the rigidity of $\mathcal{M}$, whenever $X, Y$ are fixed in $I_{F}$ with $\left.x \cdot y \in\right]-L_{F}, L_{F}$, where we always take into consideration the described working method for $\mathcal{M}$.

6. Generating a mechanical linkage $\mathcal{H}$ by coupling mechanical linkages $\mathcal{K}_{1} \otimes \cdots \otimes \mathcal{K}_{q}$, $\mathcal{A}, \mathcal{A}_{a}, \mathcal{M}$, and $\mathcal{M}_{a}$ to represent the polynomial $F$ with $V=\mathcal{L}(F)$.

The polynomial $F$ depending on the variable $x=\left(x_{1}, \ldots, x_{q}\right)$ is constructed as a mechanical linkage $\widetilde{\mathcal{H}}$ where $x$ is represented by the $q$-tuple $\left(X_{1}, \ldots, X_{q}\right)$ of vertices. For this purpose we define $V_{\text {fix }}$ of $\widetilde{\mathcal{H}}$ as the union of the sets $V_{\text {fix }}$ of every used mechanical 
linkage $\mathcal{K}_{1} \otimes \cdots \otimes \mathcal{K}_{q}, \mathcal{A}, \mathcal{A}_{a}$ and $\mathcal{M}, \mathcal{M}_{a}$ (both containing $\mathcal{M}_{C}, \mathcal{M}_{L}$ ), called sublinkages of $\widetilde{\mathcal{H}}$, where the positions of the vertices in $V_{\text {fix }}$ are cited in parts 2,4 , and 5 . The described algorithmic procedure to build up $F$ can be carried out directly, connecting the mechanical linkages $\mathcal{A}, \mathcal{A}_{a}, \mathcal{M}$, and $\mathcal{M}_{a}$.

All subpolynomials $u \in \mathcal{U}(F)$ are restricted, in particular $u(x) \in]-L_{F}, L_{F}$ [ for all $x \in B_{r+1}(0)$ which implies, by the scaling and the construction, that for $\widetilde{\mathcal{H}}$ the motion of $\left(X_{1}, \ldots, X_{q}\right)$ on $B_{r+1}(0) \subset\left(I_{F}\right)^{q}$ is parameterizing and the vertex $F\left(X_{1}, \ldots, X_{q}\right)$ gives the evaluation of $F$ on $I_{F}$ :

First take the multi-index $v$ in $\left(\mathbb{N}_{0}^{q}, \geq\right)$ proposed in part 1 to enumerate the mechanical linkages $\mathcal{A}, \mathcal{A}_{a}, \mathcal{M}$, and $\mathcal{M}_{a}$ in $\mathcal{H}$.

$\left(\mathrm{P}^{\prime}\right)$ We assume a starting realization of $\widetilde{\mathcal{H}}$ with $\left(X_{1}, \ldots, X_{q}\right)$ in $B_{r+1}(0)$, where the realizations of all sublinkages of $\widetilde{\mathcal{H}}$ are natural. Such a realization exists, since each sublinkage can be moved individually up to the required range $x \in B_{r+1}(0)$ by a parameterizing motion, where they are then connected together by their vertices $X_{1}, \ldots, X_{q}, X, Y, X+Y, X \cdot Y$. Because of (P1) and (P4) a motion of the evaluation vertices $X+Y$ or $X \cdot Y$ of two given sublinkages in $\widetilde{\mathcal{H}}$ with multi-index $v$ and $v^{\prime}$ allows the motion of $X, Y$ of a connected sublinkage with $v^{\prime \prime}>v, v^{\prime}$. By induction the $q$-tuple of vertices $\left(X_{1}, \ldots, X_{q}\right)$ can be moved on $B_{r+1}(0)$. Notice that the obtained realizations of $\widetilde{\mathcal{H}}$ need not to be regular, see $\left(\mathrm{P}^{\prime}\right)$.

$\left(\mathrm{P} 2^{\prime}\right)$ By (P2) and (P4) an induction over connected sublinkages with growing multiindex as in $\left(\mathrm{P} 1^{\prime}\right)$ ensures the rigidness of all common vertices of different sublinkages in $\widetilde{\mathcal{H}}$ if $\left(X_{1}, \ldots, X_{q}\right)$ is fixed in $B_{r+1}(0)$. So any realization of $\widetilde{\mathcal{H}}$ obtained in $\left(\mathrm{P}^{\prime}\right)$ is rigid, if $\left(X_{1}, \ldots, X_{q}\right)$ is fixed in $B_{r+1}(0)$.

$\left(\mathrm{P}^{\prime}\right)$ During the motion of $\left(X_{1}, \ldots,{\underset{\mathcal{H}}{q}}_{q}\right)$ on $B_{r+1}(0)$ described in $\left(\mathrm{P}^{\prime}\right)$, clearly each realization of all sublinkages in $\tilde{\mathcal{H}}$ is regular. Only those vertices $X_{1}, \ldots, X_{q}, X$, $Y, X+Y, X \cdot Y$ (and $Y^{\prime}, X{ }_{\lambda} Y$ for $\mathcal{M}$ and $\mathcal{M}_{a}$ ) connecting edges of two different sublinkages may cause condition (ii) of Lemma 2.6 to be injured. They are unimportant for the claimed parameterizing motion of $\left(X_{1}, \ldots, X_{q}\right)$ on $B_{r+1}(0)$ below: by induction over connected sublinkages with growing multi-index as in $\left(\mathrm{P}^{\prime}\right)$, two vertices in $X_{1}, \ldots, X_{q}, X+Y, X \cdot Y$ define the parameterization of $X, Y$ in the next connected sublinkage.

$\left(\mathrm{P}^{\prime}\right)-\left(\mathrm{P} 3^{\prime}\right)$ imply with Lemma 2.6 , that the motion of $\left(X_{1}, \ldots, X_{q}\right)$ on $B_{r+1}(0)$ is parameterizing starting with a realization of $\widetilde{\mathcal{H}}$ such that $\left(X_{1}, \ldots, X_{q}\right)$ lies in $B_{r+1}(0)$ and the realizations of all sublinkages in $\widetilde{\mathcal{H}}$ are natural. The obtained realizations are called natural realizations of $\widetilde{\mathcal{H}}$ and there is

$$
\left\{\xi \in[\tilde{\mathcal{H}}] ;\left(X_{1}, \ldots, X_{q}\right) \text { in } B_{r+1}(0)\right\} \approx B_{r+1}(0) \amalg T_{\text {rest }} .
$$

We assume natural realizations of $\underset{\mathcal{H}}{\tilde{\mathcal{H}}}$. If $\left(X_{1}, \ldots, X_{q}\right)$ is restricted in $V \subset B_{r+1}(0)$, then the vertex $F\left(X_{1}, \ldots, X_{q}\right)$ of $\widetilde{\mathcal{H}}$ representing the evaluation of $F$ is always equal to the origin of the interval $I_{F}$, since $V=\left\{\left(x_{1}, \ldots, x_{q}\right) \in \mathbb{R}^{q} ; F\left(x_{1}, \ldots, x_{q}\right)=0\right\}$. If $\left(X_{1}, \ldots, X_{q}\right)$ lies in $U(V)-V$, then the vertex $F\left(X_{1}, \ldots, X_{q}\right)$ of $\widetilde{\mathcal{H}}$ is never equal to the origin, where $U(V) \subset B_{r+1}(0)$ is an open neighborhood of $V$. These considerations 
result in

Corollary 3.5. If the vertex $F\left(X_{1}, \ldots, X_{q}\right)$ is fixed at $0 \in I_{F}$, then either

(i) $\left(X_{1}, \ldots, X_{q}\right)$ lies in $V$ assuming natural realizations only, or

(ii) $\left(X_{1}, \ldots, X_{q}\right)$ lies in $U(V)$ for realizations other than natural, or

(iii) $\left(X_{1}, \ldots, X_{q}\right)$ does not lie in $U(V)$.

Finally we obtain $\mathcal{H}$ as a mechanical linkage in the sense of Definition 2.2 by completing $V_{\text {fix }}$ of $\widetilde{\mathcal{H}}$ with $F\left(X_{1}, \ldots, X_{q}\right)$ pinned down at $0 \in I_{F}$. A realization of $\mathcal{H}$ is called natural if the corresponding realization in $\left\{\xi \in[\widetilde{\mathcal{H}}] ; F\left(X_{1}, \ldots, X_{q}\right)\right.$ at 0$\}$ is natural. By Corollaries 2.12 and 3.5 it follows that disjoint open neighborhoods in $\mathbb{R}^{2 n}$ of the sets $\left\{\xi \in[\mathcal{H}] ;\left(X_{1}, \ldots, X_{q}\right)\right.$ in $V, \xi$ natural $\}$ and its complement in $[\mathcal{H}]$ exist. So the configuration space

$$
[\mathcal{H}] \approx\left\{\xi \in[\tilde{\mathcal{H}}] ; F\left(X_{1}, \ldots, X_{q}\right) \text { at } 0\right\} \approx V \amalg T_{\text {rest }}
$$

of the mechanical linkage $\mathcal{H}$ results.

Remark 3.6. At the end we obtain a mechanical linkage $\mathcal{H}$ with some vertices $V_{\text {fix }}=$ $\left\{V_{1}, \ldots, V_{m}\right\}$ pinned down in the plane with $\operatorname{dim} \operatorname{aff}\left\{\varphi\left(V_{1}\right), \ldots, \varphi\left(V_{m}\right)\right\}=2$. Adding all edges of $\operatorname{Pot}_{2}\left(V_{\text {fix }}\right):=\left\{\left\{V_{i}, V_{j}\right\} ; V_{i}, V_{j} \in V_{\text {fix }}, i \neq j\right\}$ to the set $E$ and extending the weight function $d$ to $\operatorname{Pot}_{2}\left(V_{\text {fix }}\right)$ such that $d\left(V_{i}, V_{j}\right):=d_{\mathbb{R}}\left(p_{i}, p_{j}\right)$ for all $i, j \in\{1, \ldots, m\}$ and $i \neq j$, where $\left\{p_{1}, \ldots, p_{m}\right\}$ are the fixed points in the plane, we get an expanded mechanical linkage $\mathcal{H}_{\exp }$. If the configuration space is defined as all realizations of $\mathcal{H}_{\exp }$ in the plane modulo proper euclidean motions, then $[\mathcal{H}] \sqcup[\mathcal{H}] \approx\left(V \amalg T_{\text {rest }}\right) \amalg\left(V \amalg T_{\text {rest }}\right)$ is the configuration space of $\mathcal{H}_{\text {exp }}$. This coincides with the approach in [2]-[4], [7], and [12], but has the disadvantage that the extra edges in $\operatorname{Pot}_{2}\left(V_{\text {fix }}\right)$ are rather difficult to handle, when they are introduced from the beginning. Notice that Theorem 1.1 holds for both definitions of the configuration space.

\section{Acknowledgments}

We are grateful to Prof. P. Mani-Levitska for numerous discussions and to Prof. W. P. Thurston for a hint concerning the proof. Our thanks are due also to Prof. J.-C. Hausmann who made it possible for us to give a lecture at the Geometry Seminar in Geneva as well as to Prof. M. Kapovich and to Prof. J. Millson for reading our preprint twice, in spring 1997 and 1998, making several useful comments.

\section{References}

1. J. Bochnak, M. Coste, and M.-F. Roy, Géométrie algébrique réelle, Springer-Verlag, Berlin, 1987.

2. J.-C. Hausmann, Sur la topologie des bras articulés, Lecture Notes in Mathematics 1474, Springer-Verlag, Berlin, 1989, pp 146-159.

3. B. Jaggi, Punktmengen mit vorgeschriebenen Distanzen und ihre Konfigurationsräume, Inauguraldissertation, Universität Bern, 1992. 
4. D. Jordan, Konfigurationsräume von Gelenkmechanismen, Diplomarbeit, Universität Bern, 1997.

5. D. Jordan and M. Steiner, Configuration spaces of mechanical linkages, original version early 1997, accepted for publication in Discrete Comput. Geom., June 14, 1998, temporarily withdrawn by the authors, August 4, 1998.

6. D. Jordan and M. Steiner, Compact surfaces as configuration spaces of mechanical linkages, submitted to Israel J. Math., 1998.

7. M. Kapovich and J. Millson, On the moduli space of polygons in the Euclidean plane, J. Differential Geom. 42 (1995),133-164.

8. M. Kapovich and J. Millson, Universality theorems for configuration spaces of planar linkages, preprint, 1998.

9. H. Lebesgue, Leçons sur les constructions géométriques, Gauthier-Villars, Paris, 1950.

10. N. Mnëv, The Universality Theorems on the Classification Problem of Configuration Varieties and Convex Polytopes Varieties, Lecture Notes in Mathematics 1346, Springer-Verlag, Berlin, 1988, pp. 527-544.

11. J. Richter-Gebert, Realization Spaces of Polytopes, Lecture Notes in Mathematics 1643, Springer-Verlag, Berlin, 1996.

12. M. Steiner, Realisierungsräume von gewichteten Graphen, Diplomarbeit, Universität Bern, 1997.

Received August 26, 1997, and in revised form June 10, 1998, and February 23, 1999. 